\title{
"A QUÉ LE LLAMAN DISTANCIA, ESO ME HABRÁN DE EXPLICAR”. FRECUENCIA, CARACTERIZACIÓN GEOQUÍMICA Y CIRCULACIÓN DE LOS ARTEFACTOS DE OBSIDIANA PROVENIENTES DEL BAJO DE SARMIENTO (CHUBUT, ARGENTINA)
}

\author{
“WHAT THEY CALL DISTANCE, THAT WILL HAVE TO BE EXPLAINED TO ME”. \\ FREQUENCY, GEOCHEMICAL CHARACTERIZATION AND CIRCULATION OF \\ OBSIDIAN ARTIFACTS FROM THE SARMIENTO BASIN (CHUBUT, ARGENTINA)
}

\author{
Eduardo Moreno ${ }^{1,2}$, Santiago Peralta González ${ }^{1,2 *}$ y Alberto Enrique Pérez ${ }^{3}$
}

\begin{abstract}
Se presentan nuevos datos sobre la frecuencia, caracterización geoquímica y circulación de artefactos de obsidiana provenientes del Bajo de Sarmiento (Chubut, Patagonia argentina). Dicha región está geográficamente alejada y casi equidistante de las obsidianas de las áreas Meridional y Centro Sur de Patagonia. Se estableció que la frecuencia de obsidiana en los conjuntos es muy baja, lo que es coherente con las expectativas formuladas a partir de la relación frecuencia esperable/distancia a la fuente, y sobre la base de la estructura de los recursos líticos, que ofrece otras materias primas en las cercanías de buena calidad para la talla. El análisis geoquímico indicó una mayor frecuencia de obsidiana del área Meridional, de variedades del grupo químico PDA (Pampa del Asador) y con menor frecuencia, del área Centro-Sur. La utilización de un sistema de información geográfica permitió estimar tres tipos de distancias entre el bajo y las fuentes de obsidiana: euclidiana, caminos óptimos y rutas utilizadas en el siglo XIX según fuentes etnohistóricas. En todos los casos la fuente más cercana es PDA, en el sector Meridional. Sin embargo, se detectó la sobrerrepresentación de estas obsidianas en relación a la distancia. Esto sugiere una conexión más intensa de los cazadores recolectores del Bajo de Sarmiento con el sector Meridional.
\end{abstract}

Palabras claves: obsidiana, fuentes, circulación, Bajo de Sarmiento.

We present new data on the geochemical characterization, frequency and circulation of obsidian artifacts from the Sarmiento basin (Chubut, Argentine Patagonia). The study area is far and equidistant from known obsidian sources in southern and center-southern Patagonia. There is a low frequency of obsidian for all assemblages, coherent with expectations based on the frequency/distance to source ratio, and based on the structure of the lithic resources, which offers other good quality knapping raw materials close to the basin. The geochemical analysis indicated that the highest frequency occurs in the southern area, particularly of varieties from the Pampa del Asador (PDA), and a lesser frequency occurs in the central-south. Using the Geographic Information System (GIS), we estimated three types of distances between the basin and obsidian sources: Euclidian, optimal roads, and roads used in the 19th century according to ethnohistoric documents. In all cases, the closest source is Pampa del Asador in the southern sector. Nevertheless, the results indicate an overrepresentation of these obsidian artifacts in relation to distance. This suggests a more intense connection between the hunter-gatherers of the Sarmiento basin and the southern sector.

Key words: Obsidian, sources, circulation, Patagonia, Sarmiento basin.

El Bajo de Sarmiento se ubica en el centro sur de la Provincia de Chubut (Patagonia argentina) y consiste en una depresión de 4200 ha, con el eje mayor en sentido E-W de $70 \mathrm{~km}$ de longitud y el eje N-S con $60 \mathrm{~km}$ de extensión. Está limitado al oeste por la sierra de San Bernardo, al sur y este por la Meseta del Castillo y al norte la Sierra Corrientes y la Pampa de los Guanacos (Figura 1). Las investigaciones que se desarrollan desde hace casi 20 años han permitido identificar el uso de dicho ambiente por parte de las poblaciones cazadoras recolectoras desde al menos ca. 5000 años AP (Moreno et al. 2016; 2021). Sin

\footnotetext{
1 CONICET - Consejo Nacional de Investigaciones Científicas y Técnicas, Buenos Aires, Argentina.

2 Facultad de Humanidades y Ciencias Sociales, Universidad de la Patagonia San Juan Bosco (UNPSJB) - Instituto de Diversidad y Evolución Austral (IDEAus), CONICET. Puerto Madryn, Argentina. julianemoreno@yahoo.com; *autor crrespondiente: peraltagonzalezsantiago@gmail.com

3 Departamento de Antropología, Universidad Católica de Temuco, Temuco, Chile. aperez@uct.cl
} 




Figura 1. Área de estudio y ubicación de las fuentes de obsidiana.

Study area and location of obsidian sources.

embargo, es hacia el Holoceno Tardío final cuando, a partir de distintas líneas de investigación, pudo constatarse una mayor intensidad ocupacional evidenciada a partir de una marcada organización intrasitio (Peralta González 2020; Peralta González y Moreno 2019; Peralta González et al. 2014) en algunos casos asociados a una fuerte utilización de recursos fluviales, especialmente peces, y el desarrollo de estrategias de pesca con redes y posiblemente otras técnicas como líneas y arpones (Moreno et al. 2021; Reyes y Svoboda 2016; Svoboda 2015, 2019; Svoboda y Moreno 2018).

Los conjuntos líticos de los sitios arqueológicos del Bajo de Sarmiento, se caracterizan por la predominancia de materias primas silíceas, entre ellas el xilópalo disponible localmente (Reyes y Peralta González 2014; Reyes et al. 2013), lo cual sugiere una estrategia de aprovisionamiento orientado a rocas de disponibilidad ambiental inmediata (sensu Meltzer 1989). Sin embargo, se han registrado artefactos de obsidiana en bajas frecuencias, en sitios arqueológicos $\mathrm{y}$ en forma de hallazgos aislados, lo que resulta de gran utilidad ya que permiten hacer inferencias en relación a las estrategias de circulación y/o intercambio de esta materia prima lítica utilizada por los grupos de cazadores recolectores.
Uno de los factores que afecta a las estrategias tecnológicas está ligado al acceso a la materia prima (Bamforth 1986), y el aprovisionamiento de la misma es la actividad inicial dentro del sistema de producción lítico (Ericson 1984). Existen dos formas de planificar el aprovisionamiento directo de materia prima; integrado o "embedded" (Binford 1979) y no integrado o "disembedded" (Bamforth 1986). En la primera, el aprovisionamiento está incluido junto con otras actividades destinadas a la obtención de recursos. La segunda demanda viajes especiales destinados únicamente a la explotación de una fuente. La opción sobre qué estrategia emplear responde a distintos factores relacionados con la materia prima (disponibilidad, calidad y distribución), los recursos, la movilidad y otras actividades. Aunque un mismo grupo puede optar por distintas estrategias de acuerdo a distintas circunstancias.

En Patagonia Centro-Sur, la disponibilidad de obsidiana apta para la talla se encuentra espacialmente acotada y sus características geoquímicas han permitido identificar tres grupos: (1) tipo T/SC (subtipos 1 y 2), cuya fuente fue localizada en proximidades de Sierra Negra, al noreste de la localidad de Telsen (Provincia de Chubut) (Stern 2018; Stern et al. 2000); (2) tipo S (subtipos 1 y 2), en alrededores del Cerro Guacho 
(Pampa de Sacanana), al oeste de la localidad de Gan Gan (Provincia de Chubut) (Stern 2018; Stern et al. 2000); y un grupo químico cuya fuente aún no fue identificada, pero reconocida a partir de artefactos en la Meseta de Somuncurá y alrededores, lo que sugiere una procedencia cercana (Banegas et al. 2021; Gómez Otero y Stern 2005; Schuster et al. 2020; Stern 1999, 2018); y (3) el tipo PDA (subtipos 1, 2 a y b, y 3 a, b y c), ubicado en Pampa del Asador (Provincia de Santa Cruz) (Espinosa y Goñi 1999; Stern 1999, 2018).

En un estudio previo en el Bajo de Sarmiento, Reyes et al. (2013) analizaron $n=10$ piezas sobre un total de $\mathrm{N}=75$ artefactos de obsidiana provenientes de la cuenca del Lago Musters (Figura 1). Los criterios empleados para la elección de los elementos a analizar fueron textura, color y distribución, y a partir de ellos se buscó obtener una muestra representativa del conjunto general. Asimismo, los autores optaron por trabajar sobre desechos de talla de más de $3 \mathrm{~cm}$ y resguardar de esa manera los artefactos formatizados, dado el carácter destructivo de los análisis. Los resultados obtenidos dieron cuenta de la presencia exclusiva de obsidianas de PDA. Estos datos generaron dos nuevas preguntas:

(A) Primero, si la exclusividad detectada en el Bajo de Sarmiento no es consecuencia de un problema de muestreo. Para avanzar en este problema, se analizó la totalidad de la muestra del Lago Musters y se sumaron los hallazgos de obsidiana obtenida en Lago Colhué Huapi (Figura 1), en donde se empezó a investigar a partir del año 2014.

(B) En segundo lugar, si la tendencia detectada por Reyes et al. se mantuviera, nos proponemos indagar sobre cuáles fueron las razones por las que se optó por la fuente Meridional dada la situación equidistante de todas con respecto al Bajo de Sarmiento. Para avanzar sobre este aspecto, como alternativa a las distancias euclidianas, se estimaron las distancias mediante caminos óptimos generados en un entorno SIG y rutas indígenas relevadas en fuentes etnohistóricas para verificar si estas medidas pueden explicar la posible frecuencia de obsidiana proveniente del sector Meridional por sobre las otras fuentes.

De la amplia bibliografía existente (Banegas et al. 2021; Belardi et al. 2006; Espinosa y Goñi 1999; Molinari y Espinosa 1999; Schuster et al. 2020), se consideró el marco interpretativo desarrollado por Pallo y Borrero (2015) referido a la circulación de obsidianas desde la fuente de PDA, extendiéndose a nuestra área de estudio. Se tomaron en consideración, además de PDA, las otras fuentes conocidas (ver Pallo 2016; Stern y Franco 2000).

Por otro lado, se retoma el planteo de Pallo y Borrero (2015) que expresa que la obsidiana no formaba parte de un circuito de abastecimiento, sino que se transportaba de manera no sistemática. De este modo, se plantea que, al no haber existido sistematicidad en la circulación de obsidianas, su distribución sería un subproducto de la movilidad humana. Si la circulación humana es homogénea, la distancia es la variable que explicaría la frecuencia de estas materias primas en el bajo. Pallo y Borrero (2015) proponen que la frecuencia de obsidiana en los conjuntos líticos decrece con la distancia euclidiana indicando frecuencias superiores al 15\% a menos de $140 \mathrm{~km}$ de distancia de la fuente; entre este valor y el $1 \%$ hasta $400 \mathrm{~km}$ e inferior a $1 \%$ en sectores más alejados. Con base en este planteo proponemos como hipótesis de trabajo que la frecuencia de obsidiana presente en nuestra área de estudio sería proporcional respecto a las distancias a las fuentes de procedencia.

De esta hipótesis se desprenden dos expectativas:

(1) Las frecuencias de obsidianas en los conjuntos líticos del Bajo de Sarmiento, presentarían valores próximos al $1 \%$ dado que la distancia a las fuentes es superior a $300 \mathrm{~km}$.

(2) Si se considera la casi equidistancia del Bajo de Sarmiento a las distintas fuentes de obsidiana conocidas, es esperable que aparezcan en frecuencias similares, es decir, que no predomine un tipo por sobre el otro.

\section{Materiales y Métodos}

Para evaluar la primera expectativa se consideraron los siguientes sitios: Delta del Arroyo Vulcana 1 (DV1); Laguna de la Flecha 10 (LF10); Boliche de Jerez 3 (BJ3); Puesto Roberts 1 (PR1) y Bosque Petrificado 12 (BPR 12) (Figura 2). Los dos primeros se encuentran en el perilago de Musters y cuentan con estudios detallados del material lítico (Reyes y Peralta González 2014; Reyes et al. 2013; 2015) además de estudios tafonómicos, distribucionales, faunísticos y dataciones (Moreno y Pérez Ruíz 2010; Moreno y Svoboda 2013, 2014; Moreno et al. 2007; Peralta González 2020; Peralta González et al. 2014). BJ3 y PR1 se encuentran en el Lago Colhué Huapi. El primero no está datado, pero sí cuenta con estudios tafonómicos, distribucionales y faunísticos (Moreno et al. 2015; Peralta González 2020; Peralta González y Moreno 2019), en tanto que PR1 fue 


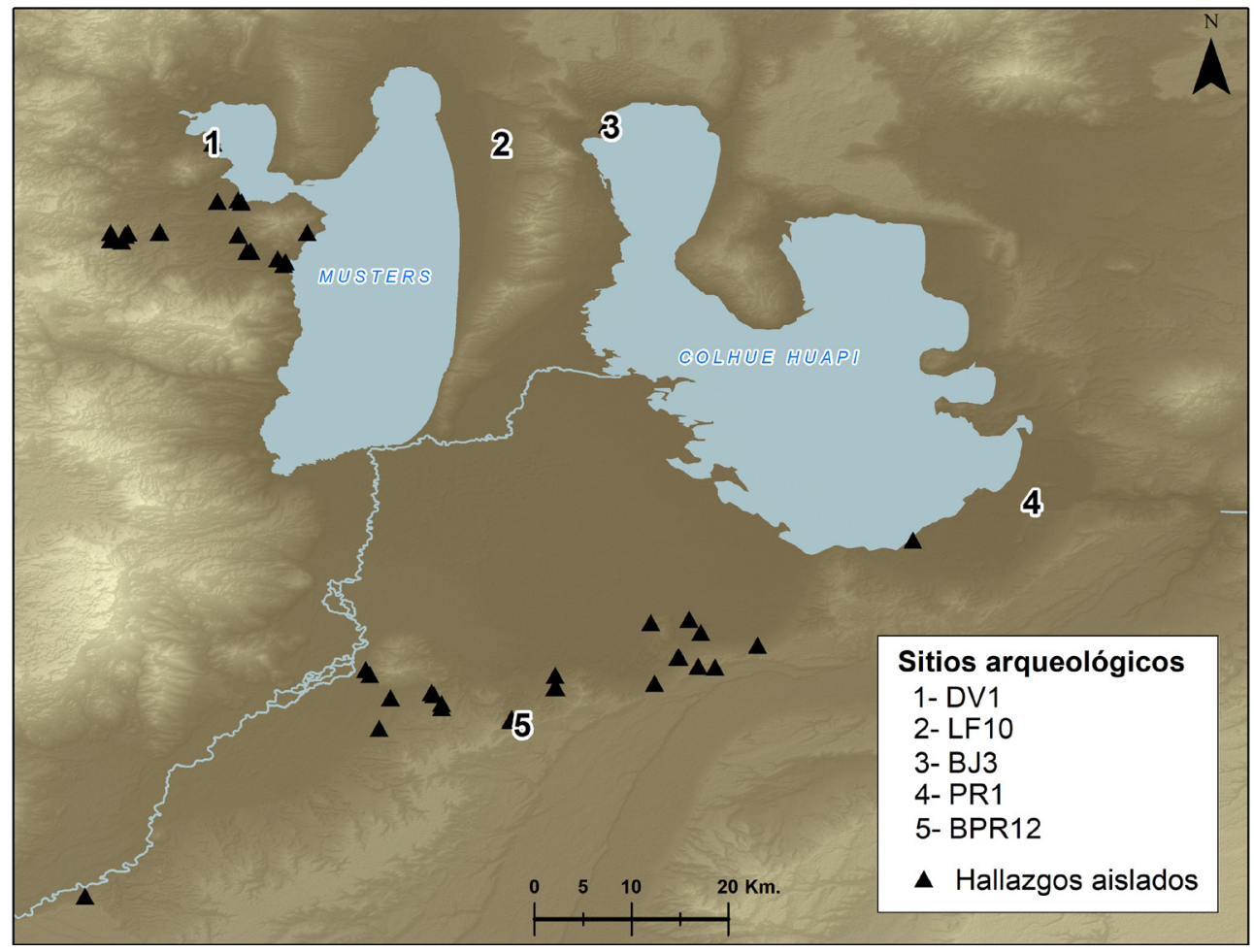

Figura 2. Sitios arqueológicos con obsidianas y hallazgos aislados.

Archaeological sites with obsidian artifacts and isolated findings.

excavado recientemente y los análisis se encuentran en desarrollo. BPR 12 se encuentra en el pedimento sur del bajo, está datado en $1230 \pm 50$ años AP y cuenta con estudios distribucionales, tafonómicos y faunísticos (Peralta González 2020). Como se señaló, BPR 12, BJ3 y PR1 no cuentan con estudios de sus contextos líticos, de modo que para este trabajo solo se analizaron las frecuencias de obsidiana versus otras rocas, sin discriminar sílices, xilópalos, basaltos, etc.

Para los estudios geoquímicos se analizaron 90 muestras de obsidiana, recolectadas en el área de estudio mediante excavaciones y recolecciones superficiales en sitios, así como artefactos aislados recolectados en prospecciones. Esta última metodología es la que permitió obtener la mayor cantidad de materiales, por lo que en muchos casos la única referencia que se cuenta es la ubicación de los artefactos de obsidiana en el terreno es decir, que en la mayor parte de los casos no se cuenta con cronología y probablemente se trate de una muestra altamente promediada. Por otro lado, si bien estas muestras seguramente fueron impactadas por coleccionistas, difícilmente estos pudieron haberlas seleccionado por tipo, ya que las distintas obsidianas son muy parecidas macroscópicamente.
La determinación geoquímica se realizó en el laboratorio de arqueología del IDEAus-CENPATCONICET, donde se instaló temporalmente un analizador portátil de fluorescencia de rayos X Tracer i5, Brucker, parte del equipamiento del Laboratorio de Cultura Material del Departamento de Antropología de la Universidad Católica de Temuco. Para el análisis se aplicó la modalidad "Obsidiana" mediante un software y calibración desarrollada por el Missouri University Reactor Research (MURR), de la Universidad de Missouri en Columbia. Se utilizó un colimador de $3 \mathrm{~mm}$ y filtro de $200 \mathrm{Cu}$, y cada exposición duró 60 segundos, es decir, el doble del tiempo sugerido por el programa, con el objetivo de minimizar el margen de error. Durante cada análisis se realizó una lectura calibrada o programada a una única fase que registró y cuantificó un total de 10 elementos pesados: titanio (Ti), manganeso $(\mathrm{Mn})$, hierro $(\mathrm{Fe})$, zinc $(\mathrm{Zn})$, galio $(\mathrm{Ga})$, rubidio $(\mathrm{Rb})$, estroncio $(\mathrm{Sr})$, circonio $(\mathrm{Zr})$, niobio $(\mathrm{Nb})$ y torio $(\mathrm{Th})$. A continuación, se procedió a establecer correlaciones entre los elementos $\mathrm{Rb}, \mathrm{Sr}$ y Zr comúnmente utilizados para discriminar grupos químicos y fuentes de procedencia (Shackley 1998), utilizando hierro $(\mathrm{Fe})$ y niobio $(\mathrm{Nb})$ como elementos 
de control para grupos químicos que presenten rangos que puedan solapar algunos de los elementos previamente mencionados.

Como se detalla más adelante, un tipo de obsidiana hallada proviene de fuentes desconocidas, pero que se ha registrado en forma de artefactos en la Meseta de Somuncurá, donde se presume su origen. A los fines de este trabajo, se agruparon todas las muestras de las fuentes de la Meseta de Somuncurá y sus proximidades en una categoría única a la que llamaremos "CentroSur" (Stern et al. 2013) para la cual se estableció un centroide entre todas las fuentes conocidas, que permitió ubicarla geográficamente y así establecer distintas mediciones de distancia respecto al Bajo de Sarmiento. El otro grupo considerado lo conforman los artefactos confeccionados sobre obsidianas provenientes de Pampa del Asador (PDA) tomando también este como una única fuente (Meridional sensu Stern 2018).

El análisis de distancia euclidiana permitió, por un lado, determinar la separación en línea recta entre el Bajo de Sarmiento y las fuentes de obsidianas, y por el otro, generar un raster que, a partir de una capa vectorial, graficó las curvas de declinación que existen entre las fuentes y el área de estudio.

Respecto a las mediciones alternativas de distancia, se consideró que la circulación implica un gasto de energía que varía en función de lo que se está transportando, de la distancia que se recorre y del terreno que se atraviesa (Binford 1980; Binford 1982; Kelly 1992, 1995) y del peso (Beck et al. 2002). Con el objetivo de pensar potenciales corredores que hayan conectado las distintas fuentes de obsidiana negra con el Bajo de Sarmiento de obsidiana, se modelaron caminos de menor costo mediante la utilización de un sistema de información geográfica (SIG). Para tal fin se consideraron como variables altura y pendiente del terreno (se utilizó un Modelo Digital de Elevación -MDE- de 30 m proveniente de Shuttle Radar Topography Mission (SRTM), disponibilidad de agua y cobertura del terreno, sobre la base de la información elaborada por el Instituto Geográfico Nacional (Argentina). Este tipo de análisis ha tenido un uso extensivo en distintas investigaciones de sociedades de cazadores recolectores de Patagonia (Franco et al. 2020; Gilio 2018; Gilio et al. 2016; Magnin 2013; Matteucci y Scheinsohn 2004; Pallo 2016; Pallo y Borrero 2020, entre otros).

El modelado de vías de circulación de menor costo fue complementado con caminos detallados en el diario del explorador galés Ap Iwan (Roberts y
Gavirati 2018), quien presenta en la cartografía de su relato caminos empleados tanto por colonos como por indígenas, los cuales fueron utilizados para circular entre las áreas consideradas en esta investigación. Se georreferenciaron dichos mapas y posteriormente se vectorizaron las rutas presentadas por el explorador, lo que permitió establecer mediciones de distancias.

Esta información se utilizó tomando en cuenta que dichos caminos no tienen por qué coincidir con los más antiguos, dado que pudieron existir cambios, no solo en los caminos sino en la forma de movilidad y requerimientos, fundamentalmente, a partir de la adopción del caballo por parte de las sociedades de cazadores recolectores. Otro tanto puede decirse de los caminos óptimos no se plantea que sean los caminos realmente utilizados, sino que se los toma como una medición alternativa a la distancia euclidiana a fin de verificar si pueden explicar las tendencias observadas.

\section{Resultados}

En los contextos líticos de los sitios BPR 12 $(\mathrm{n}=166), \mathrm{LF} 10(\mathrm{n}=239)$ y DV1 $(\mathrm{n}=773)$ no se hallaron artefactos ni desechos de talla de obsidiana (Peralta González 2020; Reyes y Peralta González 2014; Reyes et al. 2013); es de aclarar que en DV1 se halló un elemento de esta materia prima pero recolectado por fuera de los conjuntos estudiados. En BJ3 sobre un total de $\mathrm{N}=21153$ artefactos líticos, se hallaron cuatro piezas de obsidiana, es decir, un $0,02 \%$. En PR1 sobre una muestra 455 artefactos, se registraron tres piezas de obsidiana $(0,65 \%)$.

Los análisis geoquímicos realizados sobre 90 muestras mostraron una correspondencia con tres variedades de obsidiana de la fuente PDA; $\mathrm{n}=69$ (77\%) al tipo PDA 1, n=10 (11\%) al tipo PDA 2 y $\mathrm{n}=1(1 \%)$ PDA 3ab. Los resultados se completan con $\mathrm{n}=7$ (8\%) procedente de $\mathrm{S} 1$, y con $\mathrm{n}=3(8 \%)$ de MS 1. Agrupando por grandes fuentes, PDA suma 80 artefactos (\%) y las Centro Sur, 10; es decir 88,8\% contra el 11,1\% (Tabla 1 y Figuras 3 y 4).

A partir del análisis de distancias euclidianas se pudo establecer en primer lugar que el Bajo de Sarmiento está separado por 313,74 km del área Meridional y por $387,81 \mathrm{~km}$ del área Centro Sur, y en segunda instancia, se generaron buffers equidistantes de 140 km (Pallo y Borrero 2015) que permitieron representar la posición del Bajo de Sarmiento entre las dos fuentes, en intervalos de hasta $400 \mathrm{~km}$ (Figura 5).

Para evaluar potenciales vías de circulación desde el bajo hasta las áreas Centro Sur y Meridional, se 
Tabla 1. Elementos de traza presentes en las obsidianas analizadas. Trace elements present in the obsidians analyzed.

\begin{tabular}{l|c|c|c|c|c}
\hline & Código & $\begin{array}{c}\text { Grupo } \\
\text { Químico }\end{array}$ & Rb & Sr & Zr \\
\hline 1 & UCT 414 & PDA 1 & 201 & 33 & 127 \\
\hline 2 & UCT 415 & PDA 1 & 215 & 18 & 133 \\
\hline 3 & UCT 416 & PDA 1 & 187 & 30 & 116 \\
\hline 4 & UCT 417 & PDA 1 & 208 & 32 & 124 \\
\hline 5 & UCT 418 & S1 & 289 & 3 & 310 \\
\hline 6 & UCT 419 & PDA 1 & 215 & 34 & 127 \\
\hline 7 & UCT 420 & PDA 1 & 206 & 34 & 134 \\
\hline 8 & UCT 421 & PDA 1 & 209 & 32 & 133 \\
\hline 9 & UCT 422 & MS1 & 340 & 0 & 400 \\
\hline 10 & UCT 423 & MS1? & 272 & 2 & 288 \\
\hline 11 & UCT 424 & PDA 1 & 193 & 34 & 124 \\
\hline 12 & UCT 425 & PDA 1 & 213 & 35 & 136 \\
\hline 13 & UCT 426 & PDA 1 & 191 & 31 & 116 \\
\hline 14 & UCT 427 & PDA 1 & 196 & 31 & 128 \\
\hline 15 & UCT 429 & PDA 1 & 217 & 33 & 134 \\
\hline 16 & UCT 430 & PDA 2 & 252 & 2 & 126 \\
\hline 17 & UCT 431 & PDA 2 & 243 & 2 & 117 \\
\hline 18 & UCT 432 & PDA 1 & 209 & 34 & 133 \\
\hline 19 & UCT 433 & PDA 1 & 217 & 5 & 123 \\
\hline 20 & UCT 434 & PDA 1 & 209 & 34 & 125 \\
\hline 21 & UCT 435 & PDA 1 & 218 & 34 & 131 \\
\hline 22 & UCT 436 & PDA 1 & 213 & 32 & 145 \\
\hline 23 & UCT 437 & PDA 1 & 190 & 31 & 125 \\
\hline 24 & UCT 438 & PDA 1 & 206 & 33 & 130 \\
\hline 25 & UCT 439 & PDA 1 & 197 & 31 & 125 \\
\hline 26 & UCT 440 & PDA 1 & 197 & 32 & 116 \\
\hline 27 & UCT 441 & PDA 1 & 204 & 32 & 118 \\
\hline 28 & UCT 442 & PDA 1 & 205 & 34 & 133 \\
\hline 29 & UCT 443 & PDA 2 & 249 & 2 & 118 \\
\hline 30 & UCT 444 & PDA 2 & 228 & 3 & 127 \\
\hline & & &
\end{tabular}

\begin{tabular}{l|c|c|c|c|c}
\hline & Código & $\begin{array}{c}\text { Grupo } \\
\text { Químico }\end{array}$ & Rb & Sr & Zr \\
\hline 31 & UCT 445 & PDA 1 & 196 & 32 & 124 \\
\hline 32 & UCT 447 & PDA 1 & 214 & 34 & 136 \\
\hline 33 & UCT 448 & PDA 1 & 210 & 33 & 130 \\
\hline 34 & UCT 449 & PDA 2 & 229 & 4 & 131 \\
\hline 35 & UCT 450 & PDA 1 & 200 & 32 & 128 \\
\hline 36 & UCT 451 & PDA 1 & 209 & 32 & 121 \\
\hline 37 & UCT 452 & PDA 1 & 199 & 31 & 121 \\
\hline 38 & UCT 453 & PDA 1 & 205 & 33 & 139 \\
\hline 39 & UCT 454 & PDA 1 & 208 & 34 & 135 \\
\hline 40 & UCT 455 & PDA 1 & 212 & 34 & 133 \\
\hline 41 & UCT 456 & PDA 1 & 211 & 33 & 129 \\
\hline 42 & UCT 457 & PDA 1 & 225 & 36 & 139 \\
\hline 43 & UCT 458 & PDA 1 & 206 & 33 & 129 \\
\hline 44 & UCT 459 & PDA 1 & 230 & 37 & 124 \\
\hline 45 & UCT 460 & PDA 1 & 198 & 32 & 119 \\
\hline 46 & UCT 461 & PDA 1 & 223 & 35 & 132 \\
\hline 47 & UCT 462 & PDA 2 & 240 & 4 & 129 \\
\hline 48 & UCT 463 & S1 & 286 & 3 & 292 \\
\hline 49 & UCT 464 & PDA 1 & 203 & 33 & 121 \\
\hline 50 & UCT 465 & PDA 1 & 197 & 32 & 119 \\
\hline 51 & UCT 466 & PDA 1 & 207 & 32 & 133 \\
\hline 52 & UCT 467 & S1 & 285 & 3 & 288 \\
\hline 53 & UCT 468 & MS 1 & 350 & 0 & 418 \\
\hline 54 & UCT 469 & S1 & 261 & 3 & 306 \\
\hline 55 & UCT 470 & PDA 2 & 235 & 4 & 133 \\
\hline 56 & UCT 471 & PDA 1 & 203 & 33 & 142 \\
\hline 57 & UCT 472 & PDA 1 & 217 & 34 & 136 \\
\hline 58 & UCT 473 & PDA 1 & 206 & 33 & 128 \\
\hline 59 & UCT 474 & PDA 1 & 210 & 33 & 131 \\
\hline 60 & UCT 475 & PDA 1 & 204 & 32 & 122 \\
\hline & & & & \\
\hline & & & \\
\hline
\end{tabular}

\begin{tabular}{l|c|c|c|c|c}
\hline & Código & $\begin{array}{c}\text { Grupo } \\
\text { Qúmico }\end{array}$ & Rb & Sr & Zr \\
\hline 61 & UCT 476 & PDA 1 & 203 & 32 & 143 \\
\hline 62 & UCT 477 & PDA 1 & 204 & 34 & 120 \\
\hline 63 & UCT 478 & PDA 1 & 201 & 33 & 130 \\
\hline 64 & UCT 479 & PDA 1 & 208 & 32 & 124 \\
\hline 65 & UCT 480 & PDA 1 & 197 & 31 & 129 \\
\hline 66 & UCT 481 & PDA 1 & 202 & 31 & 124 \\
\hline 67 & UCT 482 & PDA 1 & 187 & 31 & 115 \\
\hline 68 & UCT 483 & PDA 2 & 256 & 5 & 142 \\
\hline 69 & UCT 484 & PDA 1 & 204 & 33 & 126 \\
\hline 70 & UCT 485 & S1 & 261 & 3 & 332 \\
\hline 71 & UCT 486 & PDA 1 & 204 & 33 & 124 \\
\hline 72 & UCT 487 & PDA 1 & 202 & 32 & 119 \\
\hline 73 & UCT 488 & PDA 1 & 217 & 35 & 141 \\
\hline 74 & UCT 489 & PDA 2 & 225 & 5 & 130 \\
\hline 75 & UCT 490 & PDA 1 & 200 & 30 & 124 \\
\hline 76 & UCT 491 & PDA 1 & 205 & 31 & 123 \\
\hline 77 & UCT 492 & PDA 1 & 184 & 29 & 118 \\
\hline 78 & UCT 493 & PDA 3ab? & 183 & 44 & 221 \\
\hline 79 & UCT 494 & S1 & 287 & 4 & 352 \\
\hline 80 & UCT 495 & PDA 1 & 211 & 35 & 125 \\
\hline 81 & UCT 496 & PDA 1 & 179 & 30 & 104 \\
\hline 82 & UCT 497 & PDA 1 & 207 & 35 & 133 \\
\hline 83 & UCT 498 & S1 & 287 & 2 & 290 \\
\hline 84 & UCT 499 & PDA 1 & 193 & 29 & 125 \\
\hline 85 & UCT 500 & PDA 2 & 228 & 1 & 115 \\
\hline 86 & UCT 502 & PDA 1 & 199 & 31 & 133 \\
\hline 87 & UCT 503 & PDA 1 & 197 & 32 & 124 \\
\hline 88 & UCT 504 & PDA 1 & 204 & 32 & 119 \\
\hline 89 & UCT 505 & PDA 1 & 202 & 31 & 124 \\
\hline 90 & UCT 506 & PDA 1 & 203 & 33 & 124 \\
\hline & & & \\
\hline 7
\end{tabular}

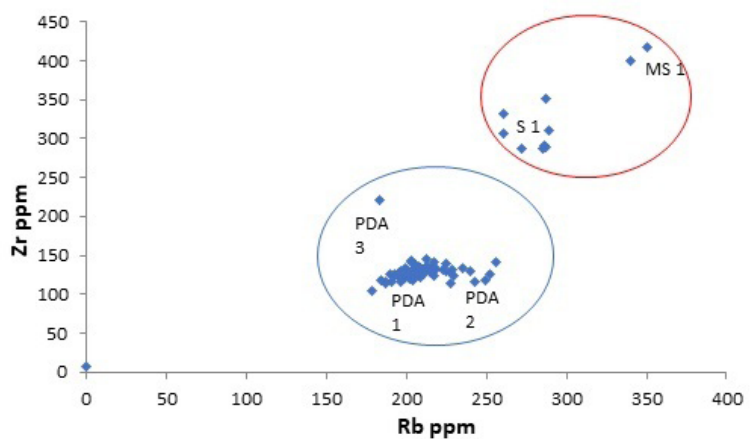

Figura 3. Zr y Rb ppm. En rojo, grupos químicos del sector Centro Sur; en azul, grupos químicos del sector Meridional.

$\mathrm{Zr}$ and $\mathrm{Rb}$ ppm. In red, chemical groups from the Central-South sector, in blue, chemical groups from the Southern sector. 


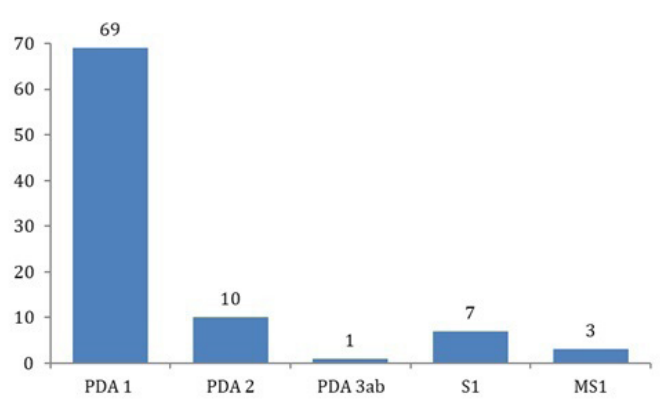

Figura 4. Grupos químicos caracterizados sobre los elementos $\mathrm{Rb}, \mathrm{Zn}, \mathrm{Sr}$.

Chemical groups characterized on the basis of elements $\mathrm{Rb}, \mathrm{Zn}, \mathrm{Sr}$.

generó un modelo de rutas a partir de la implementación de un SIG, tomando como variables la altura, pendiente, disponibilidad de agua y cobertura del terreno (Figura 6). El camino de menor costo que vincula el bajo con el área Centro Sur tiene una longitud total de 687,63 km y su recorrido rodea por el sur el Lago Colhué Huapi, luego se direcciona hasta el extremo noroeste del lago y desde allí continúa al noreste, pasando por Pampa Pelada de manera paralela al Valle del Río Chico. Luego se orienta en dirección norte hasta cruzar el Río Chubut, a pocos kilómetros de Las Plumas. Desde ese punto, el camino continúa en dirección norte hasta llegar a la fuente. Por último, la ruta que conecta el área Meridional con el Bajo de Sarmiento tiene un trayecto total de 507,94 km e inicia en PDA, en dirección noroeste hasta proximidades de Bajo Caracoles, luego de allí toma rumbo norte hasta el cañadón del Río Pinturas llegando después a cruzar el Valle del Río Deseado. Desde ese punto, retoma sentido noroeste hasta llegar al extremo sur de la Sierra San Bernardo e ingresar al bajo.

La última medición de distancias se realizó a partir de la georreferenciación de las rutas mencionadas por el explorador galés Ap Iwan (Roberts y Gavirati 2018). El camino que conecta el área Meridional con el bajo tiene un recorrido de $616,63 \mathrm{~km}$, compartiendo los primeros $200 \mathrm{~km}$ con el camino de menor costo elaborado para esta fuente, para luego dirigirse hasta el Río Mayo y desde allí tomar dirección al oeste hacia nuestra área de estudio.

En el caso del camino que une el Bajo de Sarmiento con el área Centro Sur la búsqueda en la cartografía histórica permitió detectar dos caminos que tienen como destino la Meseta de Somuncurá. Como se observa en la Figura 4, la ruta de menor costo pasaría por el medio de ambos recorridos. El primer camino, que recorre casi en su totalidad el curso del Río Chico, tiene una longitud total de $860,98 \mathrm{~km}$. El segundo cubre una distancia de 723,93 km.

\section{Discusión}

Las estrategias de circulación de obsidianas y las formas de interacción entre las sociedades que habitaron en el pasado la región patagónica solo pueden ser entendidas a partir de la integración de distintas líneas de evidencia junto con el análisis a múltiples escalas y la articulación entre regiones. Para ello, de acuerdo a lo planteado por Pallo y Borrero (2015), se postuló la hipótesis que afirma que la frecuencia de obsidiana presente en el Bajo de Sarmiento sería proporcional respecto a las distancias a las fuentes de procedencia. De esta hipótesis se desprenden dos expectativas:

La primera de ellas plantea que la frecuencia de obsidianas de cualquier fuente debería representar aproximadamente el $1 \%$ en relación con otras materias primas dentro de los conjuntos líticos en distintos sitios del Bajo de Sarmiento, ya que la distancia a las fuentes es superior a los $300 \mathrm{~km}$. Pudo verificarse la ausencia de obsidiana en los sitios BPR 12, DV1 y LF10 del Lago Musters. En el Lago Colhué Huapi se detectaron frecuencias muy bajas de obsidiana en los sitios, con valores del $0,02 \%$ en BJ3 y del $0,65 \%$ en PR1. Estos resultados permiten confirmar la hipótesis, tanto respecto a las fuentes de Patagonia Centro-Sur como de Patagonia Meridional.

La segunda expectativa proponía que al considerar la casi equidistancia del Bajo de Sarmiento a las distintas fuentes de obsidiana conocidas, era esperable que aparecieran en frecuencias similares, es decir, que no predominara un tipo por sobre el otro.

Los análisis geoquímicos sobre la muestra del bajo indican que el $88,8 \%$ de las obsidianas provienen de Pampa del Asador, en el área Meridional, mientras que solo el $11,1 \%$ corresponde a las fuentes septentrionales, es decir, una diferencia cercana a los 78 puntos porcentuales.

Si observamos en detalle las mediciones entre las fuentes de obsidiana y el Bajo de Sarmiento (Tabla 2), vemos que la mayor diferencia de distancias entre fuentes y nuestra área de estudio se da por caminos históricos, aunque no resulta considerable.

Los análisis de procedencia arrojaron una disparidad cercana al $90 \%$ en favor de la fuente Meridional, resultado que parece excesivo para que sea explicado por la diferencia en la distancia relativa entre el Bajo de Sarmiento y todas fuentes 


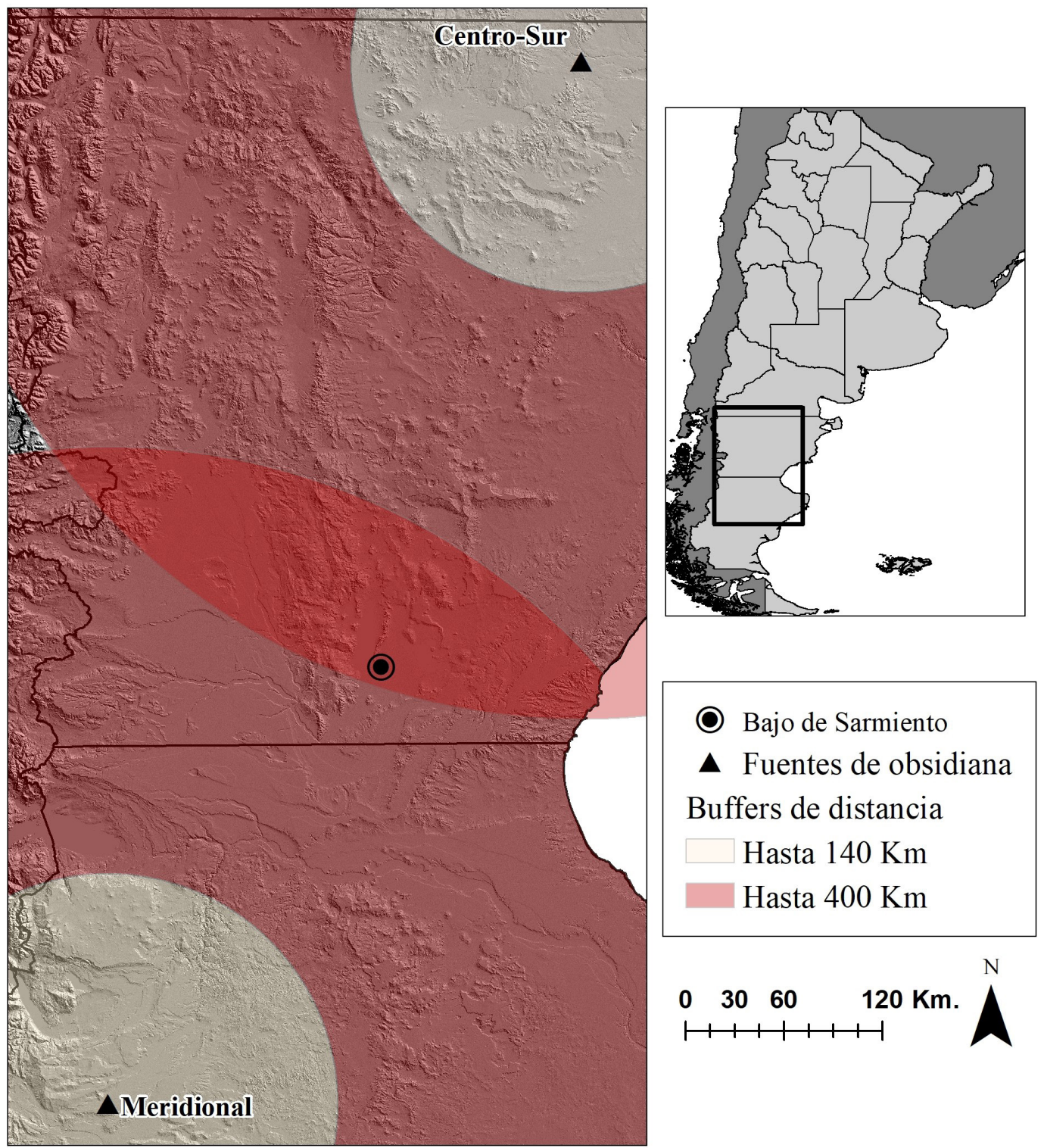

Figura 5. Buffers de distancia según Pallo y Borrero (2015).

Distance buffers according to Pallo and Borrero (2015).

que oscilan entre 15 y 29\%, según se mida por línea recta, caminos óptimos o rutas históricas.

En cuanto a su frecuencia dentro de los conjuntos líticos, la circulación de obsidiana tiene clara relación con la distancia. Sin embargo, los estudios de procedencia (geoquímicos) señalaron que la gran mayoría proviene del sector Meridional, específicamente de las diferentes variedades del grupo químico disponibles en la Pampa del Asador. Esa falta de proporción solo parece explicable por una mayor interacción humana (directa o indirecta) con el sector meridional que con el norte.

\section{Conclusión}

Este trabajo pudo determinar que la frecuencia de obsidiana dentro de los conjuntos líticos es muy escasa, por lo que su circulación está en clara relación con la distancia. Los estudios geoquímicos señalaron que hay obsidianas tanto de las fuentes Centro Sur 


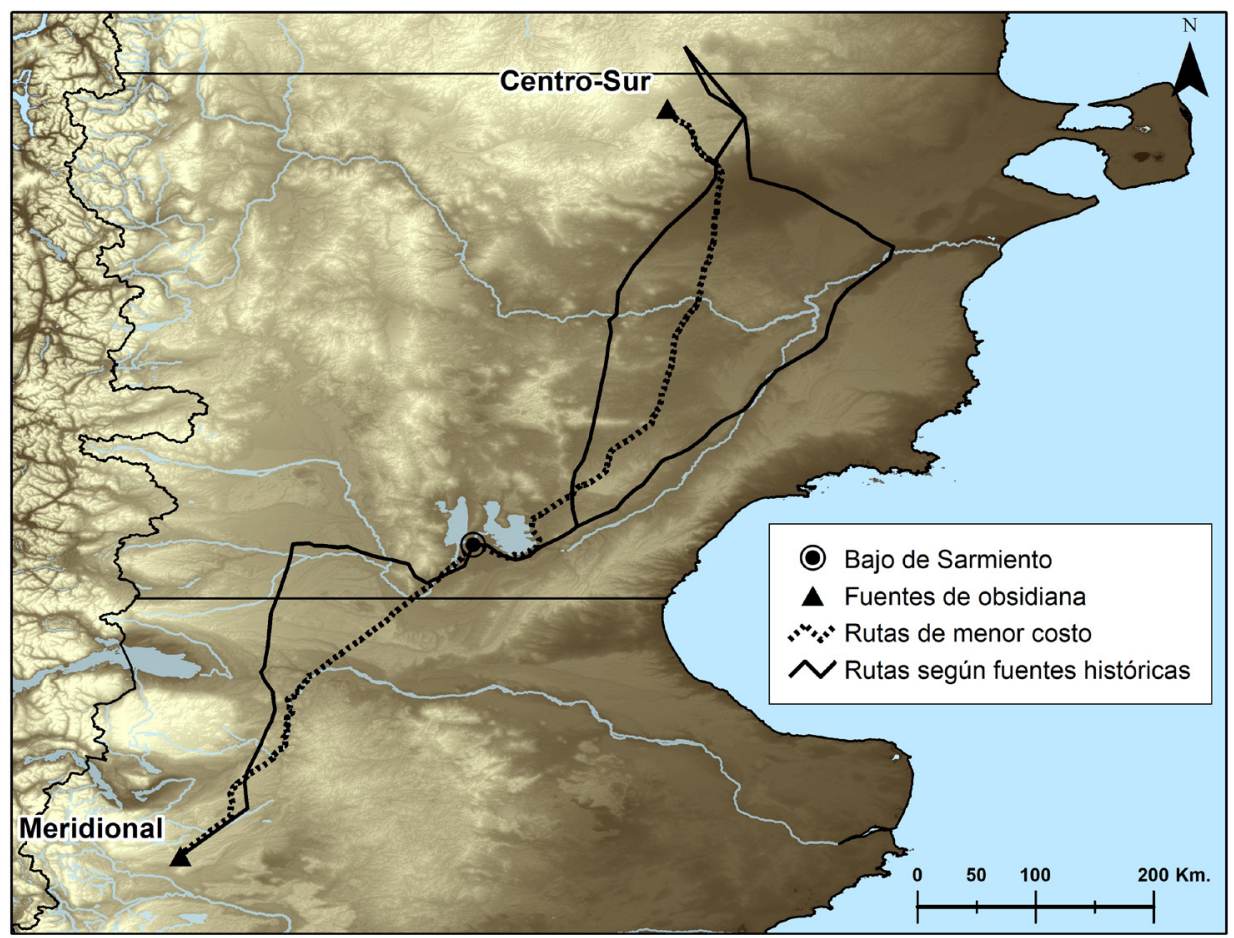

Figura 6. Caminos de menor costo y rutas mencionadas por Ip Iwan que conectan el bajo con las fuentes de obsidiana.

Lower cost roads and routes mentioned by Ip Iwan connecting the basin to obsidian sources.

Tabla 2. Distancias desde las fuentes de obsidiana al Bajo de Sarmiento.

Distances from obsidian sources to the Sarmiento basin.

\begin{tabular}{|c|c|c|c|c|}
\hline \multicolumn{5}{|l|}{ Bajo de Sarmiento } \\
\hline & Euclidiana & $\begin{array}{c}\text { Menor } \\
\text { costo }\end{array}$ & $\begin{array}{c}\text { Ruta } \\
\text { histórica } 1\end{array}$ & $\begin{array}{c}\text { Ruta } \\
\text { histórica 2 }\end{array}$ \\
\hline $\begin{array}{l}\text { Pampa del Asador } \\
\text { (Patagonia Meridional) }\end{array}$ & 313,7 & 507,9 & 616,6 & 616,6 \\
\hline $\begin{array}{l}\text { Meseta Central de } \\
\text { Chubut } \\
\text { (Patagonia Centro-Sur) }\end{array}$ & 387,8 & 687,6 & 723,9 & 860,9 \\
\hline Diferencia en km & 74,1 & 179,7 & 107,3 & 244,3 \\
\hline $\begin{array}{l}\text { Diferencia porcentual } \\
\text { en relación al camino } \\
\text { más largo }\end{array}$ & $19,1 \%$ & $26,1 \%$ & $14,8 \%$ & $28,8 \%$ \\
\hline
\end{tabular}

como Meridional, sin embargo, la gran mayoría de las obsidianas halladas provienen específicamente de las diferentes variedades del grupo químico disponibles en de Pampa del Asador. Estos resultados permiten reafirmar la tendencia observada por Reyes et al. (2013).

Al comparar la frecuencia de obsidiana de las distintas fuentes con las distancias al Bajo de Sarmiento observamos que se cumple la expectativa acerca de que es más frecuente hallar la obsidiana de la fuente más cercana. Sin embargo, en un análisis cuantitativo vemos que no hay una proporción entre la distancia y la frecuencia. Estos resultados nos permiten postular una mayor interacción social (directa o indirecta) hacia el sector Meridional que al Centro Sur. Considerando que no se observan barreras biogeográficas significativas, la explicación que se propone es que se trata de algún tipo de límite social que reduce la interacción con el norte.

Por último, es necesario a futuro explorar este posible límite considerando otras líneas de evidencia arqueológica tales como arte rupestre, tecnología lítica y cerámica. Asimismo, será necesario estudiar sus potenciales variaciones temporales, incorporando información cronológica a la distribucional.

Agradecimientos. A los dueños de las estancias donde desarrollamos las tareas de campo, Sr. Valera, Sr. Mercuri, Sr. Rua, Sr. Abait, Sr. Martínez. Agradecemos de manera muy especial al Sr. Omar Oporto de la Estancia Campanita y al Sr. Héctor Martínez de la Estancia Río Chico, quienes siempre nos reciben en sus establecimientos de la manera más hospitalaria. A colegas del Laboratorio de Arqueología y Antropología (FHyCS-UNPSJB), por sus colaboraciones en las tareas de campo. Al personal de Área Natural Protegida Bosque Petrificado Sarmiento. Agradecemos la 
colaboración de Martin Giesso. A los integrantes del Proyecto "Arqueología de las ocupaciones de cazadores recolectores del Bajo de Sarmiento" PI 1279 UNPSJB. Estos trabajos fueron financiados por el PICT 20151932 "Los cazadores recolectores en la cuenca del lago Colhué Huapi: diversificación económica, cronología de ocupación y vinculaciones con otras áreas."; el PUE- IDEAUS 22920180100012 CO "Procesos de cambio cultural y biológico en poblaciones Indígenas de Patagonia Central: estudio de casos"; IR proyectos VIP-UCT: PRO2018-AP-04, "Arqueometría y análisis de elementos aplicados en Arqueología del área centro sur de Chile", y PRO2020-AP-01, "Grupo de Arqueometría de la Universidad Católica de Temuco. Estudios funcionales físicoquímicos y biotecnológicos sobre alfarería temprana del centro sur de Chile”. Por último, agradecemos a los evaluadores de este trabajo por sus valiosos comentarios.

\section{Referencias Citadas}

Bamforth, D.B. 1986. Technological efficiency and tool curation. American Antiquity 51:38-50.

Beck, Ch., K. Taylor, G.T. Jones, C.M. Fadem, C.R. Cook y S.A. Millward 2002. Rocks are heavy: transport costs and Paleoarchaic quarry behavior in the Great Basin. Journal of Anthropological Archaeology 21:481-507.

Belardi, J.B., P. Tiberi, Ch. Stern y A. Súnico 2006. Al Este del Cerro Pampa: ampliación del área de disponibilidad de obsidiana de la Pampa del Asador (Provincia de Santa Cruz). Intersecciones en Antropología 7:27-36.

Banegas, A., J. Gómez Otero y A.E. Pérez 2021. Circulación y uso de obsidianas en la costa norte del golfo San Jorge (Prov. de Chubut, Argentina) durante el Holoceno Tardío: primeros resultados. Revista Arqueología 27 (1):193-206.

Binford, L. 1979. Organization and formation processes: Looking at curated technologies. Journal of Anthropological Research $35: 255-273$.

Binford, L. 1980. Willow smoke and dogs' tails: Hunter-gatherer settlement systems and archaeological site formation. American Antiquity 45 (1):4-20.

Binford, L. 1982. The archaeology of place. Journal of Anthropological Archaeology 1 (1):5-31.

Castro Esnal, A., Ch. Stern y C. Pérez de Micou 2017. Aplicación de estudios geoquímicos sobre artefactos de obsidiana procedentes de contextos estratigráficos y superficiales en Aldea Beleiro, SO de Chubut (Patagonia, Argentina). Magallania 45:123-135.

Ericson, D. 1992. Toward the analysis of lithic production systems. En Prehistoric Quarries Lithic Production, editado por J. Ericson y B.A. Purdy, pp 1-9. Cambridge University Press, ciudad.

Espinosa, S.L. y R.A. Goñi 1999 ¡Viven!: Una fuente de obsidiana en la provincia de Santa Cruz. Soplando en el viento. Actas de las III Jornadas de Arqueología de la Patagonia, pp. 221-234. Universidad del Comahue, Neuquén.

Franco, N., M. Glascock, B. MacDonald y B. Gilio 2020. Entre el macizo del Deseado y la margen norte de la cuenca del río Santa Cruz (Patagonia, Argentina): análisis geoquímico de artefactos de obsidiana y modelos de circulación humana. Magallania 48 (1):141-160.

Gilio, B. 2018. Modelos de caminos óptimos entre la cuenca superior y media del río Santa Cruz (Santa Cruz, Argentina). Arqueología 24 (3):143-164.

Gilio, B., F. Sarbun, y N. Franco 2016. Modelado de vías de movilidad entre sitios de cazadores-recolectores en la margen norte del río Santa Cruz (Patagonia, Argentina) durante el Holoceno tardío. Geografía y Sistemas de Información Geográfica 8:51-73.

Gómez Otero, J. y Ch. Stern 2005. Circulación, intercambio y uso de obsidianas en la costa de la provincia del Chubut (Patagonia argentina), durante el Holoceno tardío. Intersecciones 6:93-108.

Kelly, R. 1992. Mobility/Sedentism: concepts, archaeological measures and effects. Annual Review in Anthropology 21:43-66.

Kelly, R. 1995. The Foraging Spectrum. Diversity in HunterGatherer Lifeways. Smithsonian Institution Press, Washington DC.

Magnin, L. 2013. ¿Dónde pintar?: un análisis comparativo mediante SIG como aproximación a las decisiones humanas. Magallania 41 (1):193-210.

Matteucci, S.D. y V. Scheinsohn 2004. Procesamiento de imágenes, SIG y modelos ecológicos aplicados a la arqueología. GeoFocus 4:93-109.

Meltzer, D.J. 1989. Was stone exchanged among Eastern North American paleoindians? En Eastern Paleoindian Lithic Resource Use, editado por C.J. Ellis y J. Lothrop, pp. 11-39. Westview Press, Boulder.

Molinari, R. y S. Espinosa. 1999. Brilla tú, diamante "loco". Soplando en el viento. Actas de las III Jornadas de Arqueología de la Patagonia, pp. 189-198. Instituto Nacional de Antropología y Pensamiento Latinoamericano y Universidad Nacional del Comahue, Neuquén.

Moreno, E., S. Peralta González y A. Svoboda 2021. Aproximación a la cronología de la pesca en el Bajo de Sarmiento (Chubut, Argentina) a partir de la distribución de pesos líticos. Arqueología 27 (82):91-107.

Moreno, E. y H. Pérez Ruíz 2010. Evidencias de utilización prehispánica de recursos fluviales en la cuenca del Lago Musters (Chubut, Argentina). Actas del XVII Congreso Nacional de Arqueología Argentina, pp.345-350. INCIHUSA-CONICET.

Moreno, E., H. Pérez Ruíz y F. Ramírez Rozzi 2016. Esquema cronológico y evolución del paisaje en el Bajo de Sarmiento (Chubut). En Arqueología de la Patagonia. De Mar a Mar, editado por F. Mena, pp. 477-486. Ediciones CIEP / Nire Negro Ediciones, Andros Impresores, Santiago.

Moreno, E., H. Pérez Ruíz, F. Ramirez Rozzi, M. Reyes, A. Svoboda, S. Peralta González y M. Herrera Santana 2015. Primeros resultados de los trabajos arqueológicos en el lago Colhué Huapi (Chubut). Cuadernos del Instituto Nacional de Antropología y Pensamiento Latinoamericano 24 (2):133-1337.

Moreno, E y A. Svoboda 2013. Explotación de peces y guanacos en el interior de Patagonia central: aportes del sitio Delta del 
Arroyo Vulcana 1 (lago Musters, Chubut) Cazadores-recolectores del Cono Sur. Revista de Arqueología 7:49-68.

Moreno, E., B. Videla, H. Pérez Ruíz, L.S. Asencio y V. Leonforti 2007. Búsqueda de indicadores de diversificación económica prehistórica en la cuenca del Lago Musters (Chubut, Argentina). Primeros resultados. En Arqueología de Fuego-Patagonia. Levantando Piedras, Desenterrando Huesos... y Develando Arcanos, editado por F. Morello, M. Martinic, A. Prieto, G. Bahamondes, pp.22-32. CEQUA, Punta Arenas.

Pallo, M.C. 2016. Corredores naturales, fuente de obsidiana y estacionalidad: el caso de la circulación humana entre Pali Aike y sierra Baguales (Patagonia meridional). Chungara Revista de Antropología Chilena 48 (1):25-37.

Pallo, M.C. y L.A. Borrero 2015. Arqueología de corredores boscosos en Patagonia Meridional: el caso del río Guillermo. Intersecciones en Antropología 16:313-326.

Pallo, M.C. y L.A. Borrero 2020. Análisis multicriterio sobre barreras biogeográficas para la movilidad humana en Patagonia meridional. Estudios atacameños 64:277-295.

Peralta González, S. 2020. Estrategias de Uso del Espacio Intrasitio en Cazadores-Recolectores del Bajo Sarmiento durante el Holoceno Tardio (Chubut, Argentina). Tesis para optar al grado de Doctor en Arqueología, Facultad de Filosofía y Letras. Universidad de Buenos Aires.

Peralta González, S. y E. Moreno 2019. Estructuración intrasitio, registro arqueofaunístico e historia tafonómica del sitio boliche de jerez 3 (lago Colhué Huapi, Chubut). En Arqueología de la Patagonia: el Pasado en las Arenas, editado por J. Gómez Otero, A. Svoboda y A. Banegas, pp. 433-441. IDEAUS, Puerto Madryn.

Peralta González, S., J.E. Moreno y H. Pérez Ruíz 2014. La casa está en orden. Análisis espacial intrasitio de Delta del Arroyo Vulcana 1 (lago Musters, Chubut) y la movilidad de los cazadores-recolectores en Patagonia Central durante el Holoceno Tardío. Magallania 42 (2):141-153.

Reyes, M. y S. Peralta González 2014. Análisis tecno-morfológico y de materias primas de los artefactos líticos del sitio Laguna de la Flecha 10 (lago Musters, Chubut) Magallania 42 (2):199-210.

Reyes, M.R., E. Moreno y Ch. Stern 2015. Uso de obsidianas entre cazadores-recolectores en la cuenca del lago Musters (Chubut, Argentina): una aproximación desde el análisis tecnomorfológico y geoquímico. Intersecciones 16:491-495.

Reyes, M. y A. Svoboda 2016. Un acercamiento a las artes de pesca a partir del análisis de los pesos líticos en el área de los lagos Musters y Colhué Huapi (provincia de Chubut). Un acercamiento a las artes de pesca a partir del análisis de los pesos líticos en el área de los lagos Musters y Colhué Huapi (provincia de Chubut). En Arqueología de la Patagonia. De Mar a Mar, editado por F. Mena, pp. 496-509. Ediciones CIEP, Ñire Negro Ediciones, Santiago.

Reyes, M., S. Peralta González y A.L. López Ferrer 2013. Análisis preliminar de los materiales líticos del sitio Delta Vulcana 1 (Lago Musters, Chubut). En Tendencias Teórico-Metodológicas y Casos de Estudio de la Arqueología en la Patagonia, editado por A.F. Zangrando, R. Barberena, A. Gil, G. Neme, M. Giardina, L. Luna; C. Otaola, S. Paulides, L. Salgán y A. Tivoli, pp. 219226. Edición del Museo de Historia Natural de San Rafael, la SAA y el INAPL, Buenos Aires.

Roberts, T. y M. Gavirati 2018. Diarios del explorador Liwyd ap Iwan. Por las sendas tehuelches del Chubut, Santa Cruz y Aysén. Patagonia Sur Libros - La Bitácora. Puerto Madryn.

Shackley, M.S. 1998. Archaeological Obsidian Studies. Method and Theory. New York Plenum Press, London.

Schuster, V., G.I. Massaferro y A.E. Pérez 2020. Primeros análisis químicos no destructivos de obsidiana arqueológica en el valle inferior-medio del río Chubut. Patagonia Argentina. Relaciones de la Sociedad Argentina de Antropología XLV (2):447-453.

Stern, Ch. 1999. Black obsidian flrom central-south Patagonia: Chemical characteristics, sources and regional distribution of artefacts. Soplando en el viento. Actas de las III Jornadas de Arqueología de la Patagonia, pp.221-234. Instituto Nacional de Antropologia y Pensamiento Latinoamericano y Universidad Nacional de Comahue.

Stern, Ch. 2018. Obsidian sources and distribution in Patagonian, southernmost Sur America. Quaternary International 468:190-205.

Stern, Ch.R., J. Gómez Otero y J.B. Belardi 2000. Características químicas, fuentes potenciales y distribución de diferentes tipos de obsidianas en la costa e interior del centro-norte de la provincia del Chubut, Argentina. Anales del Instituto de la Patagonia (Serie Ciencias Sociales) 28:275-290.

Stern, Ch.R., A. Castro Esnal, C. Pérez de Micou, C. Méndez y F. Mena 2013. Circulación de obsidianas en Patagonia CentralSur entre $44^{\circ}$ y $46^{\circ} \mathrm{S}$. En Tendencias Teórico-Metodológicas y Casos de Estudio en la Arqueología de la Patagonia, editado por A.F. Zangrando, R. Barberena, A. Gil, G. Neme, M. Giardina, L. Luna, C. Otaola, S. Paulides, L. Salgán y A. Tivoli pp. 243250. Edición del Museo de Historia Natural de San Rafael, la SAA y el INAPL, Buenos Aires.

Stern, Ch. y N. Franco 2000. Obsidiana gris verdosa de la cuenca superior del río Santa Cruz, extremo sur de Patagonia, Anales del Instituto de la Patagonia, Serie Ciencias Humanas 28:265-73.

Svoboda, A. 2015. Los Vertebrados Pequeños en la Subsistencia de Cazadores-Recolectores: Una Evaluación Zooarqueológica Comparativa Cara Patagonia Central. Tesis para optar al grado de Doctora en Arqueología. Facultad de Filosofía y Letras, Universidad de Buenos Aires, Buenos Aires.

Svoboda, A. 2019 Ictioarqueología del sitio Boliche Jerez 3 (lago Colhué Huapi, Chubut, Argentina): implicaciones para la subsistencia de cazadores-recolectores del Holoceno tardío. Revista de Antropología del Museo de Entre Ríos 5:54-67.

Svoboda, A. y E. Moreno 2018. Peces y coipos: zooarqueología del sitio Valle Hermoso 4 (lago Colhué Huapi, Chubut). Revista del Museo de Antropología - Universidad Nacional de Córdoba 11:85-98. 